PROCEEDINGS OF THE

AMERICAN MATHEMATICAL SOCIETY

Volume 137, Number 9, September 2009, Pages 3045-3055

S 0002-9939(09)09829-3

Article electronically published on February 23, 2009

\title{
ESTIMATES FOR THE GREEN FUNCTIONS OF NONAUTONOMOUS HIGHER ORDER DIFFERENTIAL EQUATIONS
}

\author{
MICHAEL GIL' \\ (Communicated by Bryna Kra)
}

Abstract. We consider the equation

$$
\sum_{k=0}^{n} a_{k}(t) x^{(n-k)}(t)=0 \quad(t>0)
$$

where $a_{0}(t) \equiv 1 ; a_{k}(t)(k=1, \ldots, n)$ are bounded continuous functions. It is assumed that all the roots $r_{k}(t) \quad(k=1, \ldots, n)$ of the polynomial $z^{n}+$ $a_{1}(t) z^{n-1}+\ldots+a_{n}(t)$ are real for all $t \geq 0$. Sharp estimates for the Green function to the Cauchy problem and their derivatives are derived.

\section{Introduction AND STATEMENT OF THE MAIN RESUlt}

Consider the equation

$$
\sum_{k=0}^{n} a_{k}(t) x^{(n-k)}(t)=0 \quad(t>0 ; n>1),
$$

where $a_{0}(t) \equiv 1 ; a_{k}(t)(k=1, \ldots, n)$ are continuous functions defined and bounded on $[0, \infty)$. Introduce the polynomial

$$
P(z, t)=\sum_{k=0}^{n} a_{k}(t) z^{n-k} \quad(z \in \mathbb{C}, t \geq 0) .
$$

It is assumed that all the roots $r_{k}(t) \quad(k=1, \ldots, n)$ of the polynomial $P(z, t)$ for each $t \geq 0$ are real and

$$
r_{k}(t)<\gamma(t \geq 0 ; k=1, \ldots, n)
$$

with a constant $\gamma$.

In this paper we derive sharp estimates for the Green function to the Cauchy problem of equation (1.1) and their derivatives under condition (1.2). In particular, these estimates give us the conditions for exponential stability. The problem of stability analysis of various linear differential equations continues to attract the attention of many specialists despite its long history [4, 8, 12, 16, 19. It is still one of

Received by the editors August 12, 2008, and, in revised form, November 17, 2008.

2000 Mathematics Subject Classification. Primary 34A30, 34D20.

Key words and phrases. Ordinary differential equations, linear nonautonomous equations, estimates for Green functions.

This research was supported by the Kamea Fund of Israel.

(C)2009 American Mathematical Society Reverts to public domain 28 years from publication 
the most burning problems in the theory of differential equations. The basic method for the stability analysis of differential equations is the direct Liapunov method. By this method many very strong results are obtained, but finding Liapunov's functions is often connected with serious mathematical difficulties. At the same time, Theorem 1.1 gives us the exact explicit stability conditions. Moreover, to the best of our knowledge, estimates for Green's functions of higher order equations were not published in the available literature.

Recall that the Green function $G(t, \tau)$ to equation (1.1) is a function defined on $t \geq \tau \geq 0$ having continuous derivatives up to $n$-th order, satisfying (1.1) for $t>\tau$ and the initial conditions

$$
\lim _{t \downarrow \tau} \frac{\partial^{k} G(t, \tau)}{\partial t^{k}}=0 \quad(k=0, \ldots, n-2) ; \lim _{t \downarrow \tau} \frac{\partial^{n-1} G(t, \tau)}{\partial t^{n-1}}=1 .
$$

Besides, any solution of the problem

$$
\sum_{k=0}^{n} a_{k}(t) D^{n-k} v(t)=f(t) \quad\left(D:=\frac{d}{d t} ; t>0\right)
$$

with a continuous $f$ and zero initial conditions

$$
v^{(k)}(0)=0(k=0,1, \ldots, n-1)
$$

can be represented as

$$
v(t)=\int_{0}^{t} G(t, s) f(s) d s \quad(t \geq 0) .
$$

A solution to (1.1) and (1.4) is a function defined on $[0, \infty)$, having continuous derivatives up to $n$-th order, satisfying these equations for all $t>0$ and the corresponding initial conditions.

To formulate our main result, put

$$
g_{n}:=\left[\sum_{k=1}^{n}\left(C_{n}^{k}\right)^{2}-1\right]^{1 / 2} \text { and } l_{n}:=1+2 \sum_{k=1}^{n-1} \frac{g_{n}^{k} k^{k}}{e^{k}(k !)^{3 / 2}},
$$

where $C_{n}^{k}$ are the binomial coefficients. Clearly,

$$
g_{n}^{2} \leq\left(\sum_{k=0}^{n} C_{n}^{k}\right)^{2}-2=2^{2 n}-2 .
$$

Theorem 1.1. Let condition (1.2) hold. Then there is a constant $c_{0}$, such that

$$
|G(t, \tau)| \leq c_{0} \exp [\gamma(t-\tau)] \quad(t \geq \tau \geq 0) .
$$

If, in addition, $\gamma<0$, then

$$
\sup _{t \geq 0} \int_{0}^{t}|G(t, s)| d s \leq \frac{1}{|\gamma|^{n}}
$$

and

$$
\sup _{t \geq \tau \geq 0}|G(t, \tau)| \leq l_{n} \frac{|\gamma|^{n}+\zeta_{P}}{|\gamma|^{2 n-1}}
$$

where

$$
\zeta_{P}=\sup _{t \geq 0}\left[\sum_{k=0}^{n-1}\left|a_{n-k}(t) \gamma^{k}-\gamma^{n} C_{n}^{k}\right|^{2}\right]^{1 / 2} .
$$


This theorem is proved in the next two sections. It gives us the exponential stability condition $\gamma<0$. As is well known, cf. [10, 18, this condition is sharp. Namely, if (1.1) is an autonomous equation, then the inequality $\gamma<0$ is a sufficient and necessary condition for exponential stability.

Recall that A. Yu. Levin in the paper [14, Section 5] among other remarkable results had proved the following result: suppose that the $\operatorname{roots} r_{1}(t), \ldots, r_{n}(t)$ of $P(z, t)$ for each $t \geq 0$ are real and satisfy the inequalities

$$
\nu_{0} \leq r_{1}(t)<\nu_{1} \leq r_{2}(t)<\nu_{2} \leq \ldots<\nu_{n-1} \leq r_{n}(t) \leq \gamma(t \geq 0),
$$

where $\nu_{j}(j=0, \ldots, n-1)$ and $\gamma$ are constants. Then any solution $x(t)$ of (1.1) satisfies the inequality $|x(t)| \leq$ const $e^{\gamma t}(t \geq 0)$. This result is very useful for various applications; see for instance [6, 7] and the references therein. Condition (1.2) is a refinement of $(1.10)$, since $(1.10)$ does not allow the roots to intersect.

Theorem 1.1 supplements the very interesting recent investigations of the asymptotic behavior of solutions of differential equations; cf. [1, 2, 11, 13, 16. For the classical results, see [3] and the references therein.

Let us point out an explicit example which cannot be handled with the result from [14] while Theorem 1.1 can be applied.

Example 1.2. Consider the equation

$$
\frac{d^{3} x}{d t^{3}}+(2 a(t)+1) \frac{d^{2} x}{d t^{2}}+(b(t)+2 a(t)) \frac{d x}{d t}+b(t) x=0
$$

where $a(t), b(t)$ are positive continuous functions satisfying the condition

$$
a^{2}(t)>b(t), t \geq 0
$$

The variable characteristic polynomial is

$$
P(z, t)=z^{3}+(2 a(t)+1) z^{2}+(b(t)+2 a(t)) z+b(t) .
$$

The roots of this polynomial are

$$
r_{1}(t)=-a(t)+\sqrt{a^{2}(t)-b(t)}, r_{2}(t)=-a(t)-\sqrt{a^{2}(t)-b(t)}, r_{3} \equiv-1 .
$$

So the roots are real. If for some $t_{0} \geq 0$ the equality

$$
a\left(t_{0}\right)+\sqrt{a^{2}\left(t_{0}\right)-b\left(t_{0}\right)}=1
$$

holds, then we could not apply Levin's results, since $r_{2}$ and $r_{3}$ intersect. At the same time, Theorem 1.1 asserts that the considered equation is stable.

The following example shows that condition (1.2) is fulfilled in concrete situations.

Example 1.3. Consider the equation

$$
\frac{d^{3} x}{d t^{3}}+(5+b \cos (\omega t)) \frac{d^{2} x}{d t^{2}}+\left(4+(1+b \cos (\omega t))^{2}\right) \frac{d x}{d t}+\left(1+b^{2} \cos ^{2}(\omega t)\right) x=0
$$

with $\omega=$ const $\geq 0, b=$ const $>0$.

The variable characteristic polynomial is

$$
P(z, t)=z^{3}+(5+2 b \cos (\omega t)) z^{2}+\left(4+(1+b \cos (\omega t))^{2}\right) z+\left(1+b^{2} \cos ^{2}(\omega t)\right) .
$$


The roots of this polynomial are

$$
\begin{aligned}
& r_{1,2}(t)=-2-b \cos (\omega t) \pm \sqrt{(2+b \cos (\omega t))^{2}-1-b^{2} \cos ^{2}(\omega t)}= \\
& -2-b \cos (\omega t) \pm \sqrt{3+4 b \cos (\omega t)} \text { and } r_{3}=-1 .
\end{aligned}
$$

Under the condition $b<3 / 4$ all the zeros are real and by Theorem 1.1 the considered equation is stable.

\section{Proof of inequality (1.8)}

Put $R_{+}=[0, \infty)$ and introduce the space $C\left(R_{+}\right)$of real continuous and bounded on $R_{+}$functions with the sup-norm $\|v\|=\sup _{t \geq 0}|v(t)| \quad\left(v \in C\left(R_{+}\right)\right)$. We need the set

$$
\begin{aligned}
\operatorname{Dom}(L):=\left\{w \in C\left(R_{+}\right): w^{(j)} \in C\left(R_{+}\right)(j=1,2, \ldots, n),\right. \\
\\
\left.\quad w^{(k)}(0)=0(k=0,1, \ldots, n-1)\right\} .
\end{aligned}
$$

Lemma 2.1. Under the hypothesis of Theorem 1.1, let $\gamma<0$ and $f \in C\left(R_{+}\right)$. Then problem $(1.4),(1.5)$ has a unique solution $v \in \operatorname{Dom}(L)$. Moreover,

$$
\|v\| \leq \frac{\|f\|}{|\gamma|^{n}} .
$$

Proof. Define on Dom $(L)$ the operator $L$ by

$$
L u(t):=P(t, D) u=\sum_{k=0}^{n} a_{k}(t) D^{n-k} u(t) \quad(u \in \operatorname{Dom}(L)) .
$$

So problem (1.4), (1.5) can be written as $L v=f$. Since the coefficients of equation (1.4) are bounded, the roots of $P(z, t)$ are bounded on $R_{+}$. Thus,

$$
r_{k}(t) \geq-\alpha(t \geq 0 ; k=1,2, \ldots, n)
$$

for a finite positive number $\alpha$. Define on $\operatorname{Dom}(L)$ also the operator $L_{0}$ by

$$
L_{0} u(t):=(D+\alpha)^{n} u(t)=\left(\frac{d}{d t}+\alpha\right)^{n} u(t) .
$$

Then the inverses to $L$ and $L_{0}$ satisfy the relations

$$
L^{-1}=L_{0}^{-1} L_{0} L^{-1}=L_{0}^{-1}\left(L L_{0}^{-1}\right)^{-1} .
$$

Below we check that the inverses really exist.

By the Laplace transform for any $y \in C\left(R_{+}\right)$we have

$$
L_{0}^{-1} y(t)=\frac{1}{2 \pi i} \int_{-i \infty}^{i \infty} \frac{e^{\lambda t} \tilde{y}(\lambda)}{(\lambda+\alpha)^{n}} d \lambda,
$$

where $\tilde{y}$ is the Laplace transform to $y$. Set $h:=L L_{0}^{-1} y$. Then

$$
h(t)=\frac{1}{2 \pi i} \int_{-i \infty}^{i \infty} \frac{e^{\lambda t} P(\lambda, t) \tilde{y}(\lambda) d \lambda}{(\lambda+\alpha)^{n}} .
$$

Hence,

$$
h(t)=\frac{1}{2 \pi i} \int_{-i \infty}^{i \infty} e^{\lambda t} \tilde{y}(\lambda) \prod_{k=1}^{n} \frac{\lambda-r_{k}(t)}{\lambda+\alpha} d \lambda .
$$


Put

$$
F(t, \nu)=\frac{1}{2 \pi i} \int_{-i \infty}^{i \infty} e^{\lambda t} \tilde{y}(\lambda) \prod_{k=1}^{n} \frac{\lambda-r_{k}(\nu)}{\lambda+\alpha} d \lambda(t, \nu \geq 0)
$$

Thus

$$
F(t, t)=h(t)
$$

We can write

$$
F(t, \nu)=\frac{1}{2 \pi i} \int_{-i \infty}^{i \infty} e^{\lambda t} \tilde{y}(\lambda) \prod_{k=1}^{n}\left(1-\frac{\alpha+r_{k}(\nu)}{\lambda+\alpha}\right) d \lambda(t, \nu \geq 0) .
$$

Then by the convolution property,

$$
F(t, \nu)=\left(K_{1}(\nu) * K_{2}(\nu) * \ldots * K_{n}(\nu) * y\right)(t),
$$

where for a $u \in C\left(R_{+}\right)$,

$$
\left(K_{j}(\nu) * u\right)(t)=u(t)-\left(\alpha+r_{j}(\nu)\right) \int_{0}^{t} e^{-\alpha(t-s)} u(s) d s,
$$

since $e^{-\alpha t}$ is the Laplace original to $(\lambda+\alpha)^{-1}$. So

$$
\left(K_{j}(t) * u\right)(t)=u(t)-\left(\alpha+r_{j}(t)\right) \int_{0}^{t} e^{-\alpha(t-s)} u(s) d s .
$$

Thus with $\beta=-\gamma$ we have

(2.4) $\sup _{t \geq 0}\left|\left(K_{j}(t) * u\right)(t)\right| \geq\|u\|-(\alpha-\beta)\|u\| \sup _{t \geq 0} \int_{0}^{t} e^{-\alpha(t-s)} d s=\|u\|\left(1+\frac{\beta-\alpha}{\alpha}\right)$

since $-r_{j}(\nu)>\beta(\nu \geq 0)$. From (2.3) it follows that

$$
h(t)=F(t, t)=\left(K_{1}(t) * K_{2}(t) * \ldots * K_{n}(t) * y\right)(t) .
$$

Put

$$
y_{j}(t)=\left(K_{j+1}(t) * \ldots * K_{n}(t) * y\right)(t) \quad(j=1, \ldots, n-1) .
$$

Then

$$
h(t)=\left(K_{1}(t) * y_{1}\right)(t)=y_{1}(t)-\left(\alpha-r_{1}(t)\right) \int_{0}^{t} e^{-\alpha(t-s)} y_{1}(s) d s
$$

and

$$
y_{j}(t)=\left(K_{j+1}(t) * y_{j+1}\right)(t)(j=1, \ldots, n-1) ; y_{n}=y
$$

By (2.4) we have

Thus,

$$
\left\|y_{j}\right\| \geq\left\|y_{j+1}\right\| \frac{\beta}{\alpha}
$$

$$
\|y\| \leq\left\|y_{n-1}\right\| \frac{\alpha}{\beta} \leq\left\|y_{n-2}\right\| \frac{\alpha^{2}}{\beta^{2}} \leq \ldots \leq\left\|y_{1}\right\| \frac{\alpha^{n-1}}{\beta^{n-1}} \leq\|h\| \frac{\alpha^{n}}{\beta^{n}} .
$$

This means that the operator $T:=L L_{0}^{-1}$ satisfies the inequality

$$
\|T y\| \frac{\alpha^{n}}{\beta^{n}} \geq\|y\| \text {. }
$$

Let us prove that $T$ is invertible. Indeed, from (2.2), by the convolution property we obtain that

$$
h(t)=F(t, t)=y(t)+\int_{0}^{t} W(t, t-s) y(s) d s
$$


where

$$
W(\nu, t)=-\frac{1}{2 \pi i} \int_{-i \infty}^{i \infty} e^{\lambda t} \frac{(\lambda+\alpha)^{n}-P(\lambda, \nu)}{(\lambda+\alpha)^{n}} d \lambda(\nu \geq 0) .
$$

Furthermore, let $C(0, \tau)$ be the Banach space of functions continuous on $[0, \tau]$ with a positive $\tau<\infty$. Introduce on $C(0, \tau)$ the Volterra operator $V$ by

$$
(V w)(t)=\int_{0}^{t} W(t, t-s) w(s) d s .
$$

Then $y-V y=T y=h$. By the Neumann series,

$$
T^{-1}=(I-V)^{-1} h=\sum_{k=0}^{\infty} V^{k} .
$$

Here $I$ is the unit operator. Note that the Neumann series of any Volterra operator with a continuous kernel converges in the sup-norm on each finite segment, since the spectral radius of that operator in a space of continuous functions defined on a finite segment is equal to zero. So (2.5) implies the inequality

$$
\left\|T^{-1}\right\|=\left\|\left(L L_{0}^{-1}\right)^{-1}\right\| \leq \frac{\alpha^{n}}{\beta^{n}} .
$$

Furthermore, take into account that

$$
L_{0}^{-1} y(t)=\frac{1}{2 \pi i} \int_{-i \infty}^{i \infty} \frac{e^{\lambda t} \tilde{y}(\lambda)}{(\lambda+\alpha)^{n}} d \lambda=\int_{0}^{t} Q(t-s) y(s) d s \quad\left(y \in C\left(R_{+}\right)\right),
$$

where

$$
Q(t)=\frac{1}{2 \pi i} \int_{-i \infty}^{i \infty} \frac{e^{\lambda t}}{(\lambda+\alpha)^{n}} d \lambda .
$$

By the Cauchy formula for derivatives, we have

$$
Q(t)=\frac{t^{n-1}}{(n-1) !} e^{-\alpha t}(t \geq 0) .
$$

Hence,

$$
\begin{aligned}
\left\|L_{0}^{-1} y\right\| & =\sup _{t \geq 0}\left|\int_{0}^{t} Q(t-s) y(s) d s\right| \leq\|y\| \sup _{t \geq 0} \int_{0}^{t} \frac{(t-s)^{n-1}}{(n-1) !} e^{-\alpha(t-s)} d s \\
& =\|y\| \int_{0}^{\infty} \frac{s^{n-1}}{(n-1) !} e^{-\alpha s} d s=\|y\| \frac{1}{\alpha^{n}}\left(y \in C\left(R_{+}\right)\right) .
\end{aligned}
$$

So

$$
\left\|L_{0}^{-1}\right\| \leq \frac{1}{\alpha^{n}}
$$

Now (2.1) and (2.6) imply

$$
\left\|L^{-1}\right\| \leq\left\|L_{0}^{-1}\right\|\left\|\left(L L_{0}^{-1}\right)^{-1}\right\| \leq \frac{1}{\beta^{n}} .
$$

Since $\beta=|\gamma|$, this proves the required result.

Note that a result similar to Lemma 2.1 was proved in [9], but the proof in that paper contains a mistake.

Inequality (1.8) is due to (1.6) and Lemma 2.1. 


\section{Proofs of inequalities (1.7) And (1.9)}

Again take $\gamma<0$. Clearly,

$$
Q^{(j)}(t)=e^{\gamma t} \sum_{k=0}^{j} C_{j}^{k} \frac{t^{n-1-k}}{(n-k-1) !} \gamma^{j-k} \quad(j=1,2, \ldots) .
$$

Recall that $C_{j}^{k}$ are the binomial coefficients. Clearly, $Q(t)$ is a solution of the equation

$$
(D-\gamma)^{n} z=0
$$

with the initial conditions

$$
Q^{(j)}(0)=0(j=0, \ldots, n-2), \quad Q^{(n-1)}(0)=1 .
$$

Since

$$
\max _{t \geq 0} e^{\gamma t} t^{k}=\frac{1}{|\gamma|^{k}} \max _{s \geq 0} e^{-s} s^{k}=\frac{\nu_{k}}{|\gamma|^{k}}, \text { where } \nu_{k}=k^{k} e^{-k},
$$

we have by (3.1),

$$
\left\|Q^{(j)}\right\| \leq m_{j}(\gamma), \text { where } m_{j}(\gamma):=\sum_{k=0}^{j} C_{j}^{k} \frac{\nu_{n-1-k}}{|\gamma|^{n-j-1}(n-k-1) !} .
$$

In particular,

$$
\|Q\| \leq m_{0}(\gamma)=\frac{|\gamma|^{-n+1} \nu_{n-1}}{(n-1) !}=e^{-(n-1)} \frac{(n-1)^{n-1}}{(n-1) !|\gamma|^{n-1}} .
$$

Furthermore, subtract (3.2) from (1.1) and put $v(t)=x(t)-z(t)$, where $x$ is a solution of (1.1) and $z$ is a solution of (3.2) with the same initial conditions:

$$
z^{(j)}(0)=x^{(j)}(0)(j=0, \ldots, n-1) .
$$

Then

$$
0=P(D, t) x(t)-(D-\gamma)^{n} z(t)=P(D, t) v(t)+\left[P(D, t)-(D-\gamma)^{n}\right] z(t) .
$$

Thus $v$ satisfies problem (1.4), (1.5) with

$$
\begin{aligned}
f(t) & =-\left[P(D, t)-(D-\gamma)^{n}\right] z(t)=-\sum_{j=1}^{n}\left(a_{n-j}(t)-C_{n}^{j} \gamma^{n-j}\right) D^{j} z(t) \\
& =-\sum_{j=0}^{n-1}\left(a_{n-j}(t) \gamma^{j}-C_{n}^{j} \gamma^{n}\right) \gamma^{-j} D^{j} z(t) .
\end{aligned}
$$

Hence, by the Schwarz inequality,

$$
|f(t)|^{2} \leq \sum_{j=0}^{n-1}\left|a_{n-j}(t) \gamma^{j}-C_{n}^{j} \gamma^{n}\right|^{2} N_{\gamma}^{2}(t) \quad(t \geq 0),
$$

where

$$
N_{\gamma}(t)=\left[\sum_{k=0}^{n-1}\left|\gamma^{-k} z^{(k)}(t)\right|^{2}\right]^{1 / 2}
$$

Thus

$$
|f(t)| \leq \zeta_{P} N_{\gamma}(t) \quad(t \geq 0) .
$$


Substitute $s=-t \gamma$ into (3.2). Then

$$
(d / d t-\gamma)^{n} z(t)=|\gamma|^{n}(d / d s+1)^{n} w(s)=0,
$$

where $w(s)=z(s /|\gamma|)$. Rewrite the equation $(d / d s+1)^{n} w(s)=0$ in the vector form

$$
d \tilde{w}(s) / d s=B \tilde{w}(s)
$$

with the constant matrix

$$
B=\left(\begin{array}{ccccc}
-C_{n}^{1} & -C_{n}^{2} & \ldots & -C_{n}^{n-1} & -1 \\
1 & 0 & \ldots & 0 & 0 \\
0 & 1 & \ldots & 0 & 0 \\
. & . & \ldots & . & . \\
0 & 0 & \ldots & 1 & 0
\end{array}\right)
$$

and $\tilde{w}(s)=$ column $\left(w(s), \ldots, w^{(n-1)}(s)\right)$. Taking the Euclidean norm

$$
\|\tilde{w}(s)\|_{R^{n}}=\left[\sum_{k=0}^{n-1}\left|w^{(k)}(s)\right|^{2}\right]^{1 / 2},
$$

we have

$$
\|\tilde{w}(s)\|_{R^{n}} \leq\left\|e^{-B s}\right\|_{R^{n}}\|\tilde{w}(0)\|_{R^{n}} .
$$

By Theorem 2.7.1 from [5],

$$
\left\|e^{B s}\right\|_{R^{n}} \leq e^{-s} \sum_{k=0}^{n-1} \frac{g_{n}^{k} s^{k}}{(k !)^{3 / 2}} \quad(s \geq 0) .
$$

Taking into account that

$$
\max _{s} e^{-s} \sum_{k=0}^{n-1} \frac{g_{n}^{k} s^{k}}{(k !)^{3 / 2}} \leq \sum_{k=0}^{n-1} \frac{g_{n}^{k} \nu_{k}}{(k !)^{3 / 2}}=\sum_{k=0}^{n-1} \frac{g_{n}^{k}}{(k !)^{3 / 2}} k^{k} e^{-k}=l_{n}
$$

we get

$$
\left\|e^{-s B}\right\|_{R^{n}} \leq e^{-s} \sum_{k=0}^{n-1} \frac{g_{n}^{k} s^{k}}{(k !)^{3 / 2}} \leq l_{n} \quad(s \geq 0) .
$$

So

$$
\|\tilde{w}(s)\|_{R^{n}} \leq l_{n}\|\tilde{w}(0)\|_{R^{n}} \quad(s \geq 0) .
$$

But $s=|\gamma| t$ and

$$
\frac{d^{k} z(t)}{d t^{k}}=|\gamma|^{k} \frac{d^{k} w(s)}{d s^{k}}
$$

and thus, $\|\tilde{w}(s)\|_{R^{n}}=N_{\gamma}(t)$. Now (3.7) implies $N_{\gamma}(t) \leq l_{n} N_{\gamma}(0)(t \geq 0)$. Hence by (3.6) we get the inequality $\|f\| \leq \zeta_{P} l_{n} N_{\gamma}(0)$. Making use of Lemma 2.1, we arrive at the inequality $\|v\| \leq 2\|f\| /|\gamma|^{n}$. Thus $\|x\| \leq\|z\|+\|f\| /|\gamma|^{n}$. But $|z(t)| \leq N_{\gamma}(t)$ $(t \geq 0)$ and according to $(3.7), N_{\gamma}(t) \leq l_{n} N_{\gamma}(0), t \geq 0$. Consequently,

$$
\|x\| \leq l_{n} N_{\gamma}(0)\left[1+\zeta_{P} /|\gamma|^{n}\right] .
$$

Taking the initial conditions (1.3) with $\tau=0$, we get $N_{\gamma}(0)=1 /|\gamma|^{n-1}$ and

$$
\|G(t, 0)\| \leq l_{n} \frac{|\gamma|^{n}+\zeta_{P}}{|\gamma|^{2 n-1}} .
$$

Now, replacing $t$ by $t-\tau$, we arrive at (1.9). 
Now let $\gamma$ be arbitrary. To prove inequality (1.7), put in (1.1)

$$
x(t)=y(t) \exp [b t]
$$

with a real constant $b$. Evidently,

$$
\sum_{k=0}^{n} a_{n-k}(t) D^{k} e^{b t} y=e^{b t} \sum_{k=0}^{n} a_{n-k}(t) \sum_{j=0}^{k} C_{k}^{j} b^{k-j} D^{j} y=e^{b t} \sum_{k=0}^{n} a_{n-k}(t)(D+b)^{k} y .
$$

So $y$ satisfies the equation

$$
P(D+b, t) y=0 .
$$

Take $b=\gamma+\epsilon$ with a positive $\epsilon$ small enough. Under (1.2) the roots $\tilde{r}_{j}(t)$ of $P(z+b, t)$ satisfy the inequality $\tilde{r}_{j}(t) \leq \gamma-b=-\epsilon$. Inequality (1.9) asserts that the Green function $G_{b}$ to equation (3.10) is bounded:

$$
\sup _{t \geq 0}\left|G_{b}(t, 0)\right| \leq d(\epsilon)=\text { const. }
$$

Now (3.9) implies $|G(t, 0)| \leq d(\epsilon) \exp [(\gamma+\epsilon) t]$. Hence replacing $t$ by $t-\tau$ we arrive at the inequality

$$
|G(t, \tau)| \leq d(\epsilon) \exp [(\gamma+\epsilon)(t-\tau)](t \geq \tau \geq 0) .
$$

Since $\epsilon$ is arbitrary, we get (1.7). The theorem is proved.

\section{Derivatives of the Green function}

Let $f^{(k)} \in C\left(R_{+}\right)(k=0, \ldots, n)$. Recall the Kolmogorov inequalities

$$
\left\|f^{(j)}\right\| \leq \kappa_{j}\left\|f^{(n)}\right\|^{j / n}\|f\|^{1-j / n}(j=1, \ldots, n-1),
$$

where $\kappa_{j}=\kappa_{n j}$ are the Kolmogorov constants; cf. [15, p. 7]. Denote by $\tilde{z}(P)$ the unique positive root of the algebraic equation

$$
y^{n}=\sum_{j=0}^{n-1}\left\|a_{n-j}\right\| \kappa_{j} y^{j}
$$

Lemma 4.1. Let equation (1.1) have a solution $x \in C\left(R_{+}\right)$. Then $\left\|x^{(n)}\right\| \leq$ $\|x\| \tilde{z}^{n}(P)$.

Proof. By (1.1) and (4.1),

$$
\left\|x^{(n)}\right\| \leq \sum_{k=0}^{n-1}\left\|a_{n-k}\right\| \kappa_{k}\|x\|^{1-k / n}\left\|x^{(n)}\right\|^{k / n} .
$$

Then with $\tilde{y}=\left(\left\|x^{(n)}\right\| /\|x\|\right)^{1 / n}$, we have

$$
\tilde{y}^{n} \leq \sum_{k=0}^{n-1}\left\|a_{n-k}\right\| \kappa_{k} \tilde{y}^{k}
$$

This proves the lemma.

The Kolmogorov inequalities (4.1) and the previous lemma imply

Corollary 4.2. Let equation (1.1) have a solution $x \in C\left(R_{+}\right)$. Then $\left\|x^{(j)}\right\| \leq$ $\kappa_{j} \tilde{z}^{j}(P)\|x\|(j=1, \ldots, n)$. 
Put

$$
w(P):=2 \max _{j=1, \ldots, n} \sqrt[j]{\left\|a_{j}\right\| \kappa_{n-j}} .
$$

By Corollary 18.2 in $[7$ the inequality $\tilde{z}(P) \leq w(P)$ holds. Thus, the following result is valid.

Corollary 4.3. Let equation (1.1) have a solution $x \in C\left(R_{+}\right)$. Then $\left\|x^{(j)}\right\| \leq$ $\kappa_{j} w^{j}(P)\|x\|(j=1, \ldots, n)$.

Now (1.9) yields the following result.

Corollary 4.4. Let condition (1.2) hold. Then

$$
\sup _{t \geq \tau \geq 0}\left|\frac{\partial^{j} G(t, \tau)}{\partial t^{j}}\right| \leq \theta(\gamma) \kappa_{j} \tilde{z}^{j}(P) \leq \theta(\gamma) \kappa_{j} w^{j}(P) \quad(j=1, \ldots, n)
$$

where

$$
\theta(\gamma):=l_{n} \frac{|\gamma|^{n}+\zeta_{P}}{|\gamma|^{2 n-1}}
$$

\section{REFERENCES}

[1] Belaïdi, B., Estimation of the hyper-order of entire solutions of complex linear ordinary differential equations whose coefficients are entire functions. Electron. J. Qual. Theory Differ. Equ., vol. 2002, Paper No. 5, electronic only (2002), 1-8. MR1895276 (2003a:34149)

[2] Caraballo, T., On the decay rate of solutions of non-autonomous differential systems, Electron. J. Diff. Eqns., vol. 2001, No. 5 (2001), 1-17. MR.1811778 (2001m:34115)

[3] Chicone, C., Ordinary Differential Equations with Applications. 2nd ed., Texts in Applied Mathematics, 34, Springer, New York, 2006. MR2224508 (2006m:34001)

[4] De la Sen, M., Robust stability of a class of linear time-varying systems. IMA J. Math. Control Inf. 19, No. 4 (2002), 399-418. MR.1949011 (2003j:93060)

[5] Gil', M.I., Operator Functions and Localization of Spectra, Lecture Notes in Mathematics, vol. 1830, Springer-Verlag, Berlin, 2003. MR2032257 (2005f:47036)

[6] Gil', M.I., A new stability test for nonlinear nonautonomous systems, Automatica J. IFAC 40 (2004), 2161-2165 (2005). MR2156162 (2006d:93113)

[7] Gil', M.I., Explicit Stability Conditions for Continuous Systems, Lectures Notes in Control and Information Sci., Vol. 314, Springer-Verlag, Berlin, 2005. MR.2133008 (2006b:93170)

[8] Gil', M.I., Stability of nonlinear systems with differentiable linear parts, Circuits, Systems and Signal Processing 24, No. 3 (2005), 243-251. MR2168010 (2007a:34079)

[9] Gil', M.I., Liapunov exponents for higher-order linear differential equations whose characteristic equations have variable real roots, Electronic Journal of Differential Equations, vol. 2008, No. 54 (2008), 1-6. MR.2392958

[10] Harris, C.J. and Miles, J.F., Stability of Linear Systems, Academic Press, London-New York, 1980. MR0662825 (83m:34041)

[11] Hoang Nam, The central exponent and asymptotic stability of linear differential algebraic equations of index 1. Vietnam J. Math. 34, No. 1 (2006), 1-15. MR2233315 (2007e:34006)

[12] Hovhannisyan, G.R., Asymptotic stability for second-order differential equations with complex coefficients, Electron. J. Diff. Eqns., Vol. 2004, No. 85 (2004), 1-20. MR2075424 (2005f:34141)

[13] Illarionova, O.G., On the stability of the $k$ th general exponent of a linear system of differential equations. Differ. Equations 32, No. 9 (1996), 1173-1176; translation from Differ. Uravn. 32, No. 9 (1996), 1171-1174. MR1600828 (99e:34061)

[14] Levin, A. Yu., The non-oscillation of solutions of the equation $x^{(n)}+p_{1}(t) x^{(n-1)}(t)+\ldots+$ $p_{n}(t) x=0$, Russian Mathematical Surveys, 24(2) (1969), 43-99. MR0254328 (40:7537)

[15] Mitrinović, D.S., Pečarić, J.E. and Fink, A.M., Inequalities Involving Functions and Their Integrals and Derivatives, Kluwer Academic Publishers, Dordrecht, 1991. MR1190927 (93m:26036) 
[16] Morozov, O.I., A criterion for upper semistability of the highest Lyapunov exponent of a nonhomogeneous linear system. Differ. Equations 28, No. 4 (1992), 473-478; translation from Differ. Uravn. 28, No. 4 (1992), 587-593. MR.1188510 (93f:34080)

[17] Prësdorf, Z., Linear Integral Equations. Current Problems in Mathematics, vol. 27, 5-130, Akad. Nauk SSSR, Moscow, 1988 (in Russian). MR.1178114

[18] Rugh, W.J., Linear System Theory. Prentice Hall, Englewood Cliffs, New Jersey, 1993. MR.1211190 (94b:93002)

[19] Tunç, C., Stability and boundedness of solutions to certain fourth-order differential equations, Electron. J. Diff. Eqns., Vol. 2006, No. 35 (2006), 1-10. MR2213579(2007a:34085)

Department of Mathematics, Ben Gurion University of the Negev, P.O. Box 653, BeER-SHeva 84105, ISRAEL

E-mail address: gilmi@cs.bgu.ac.il 